\title{
Entlastung für das Pflegepersonal im Operationssaal
}

\author{
S. Rübenacker
}

Cave, die Lagerungshilfe nur kurzzeitig während der Desinfektion der Haut anwenden - ansonsten Thrombosegefahr! Die Schriftleitung

\section{Zusammenfassung}

In kleineren und mittleren Krankenhäusern ist die Personalsituation oftmals äußerst angespannt. Personalberechnungen basieren auf lange zurück liegende Arbeitsplatzanalysen, die den heutigen Bedürfnissen nicht gerecht werden. Gut ausgebildetes und hochqualifiziertes Personal muss Tätigkeiten verrichten, die nicht ihren Fähigkeiten entsprechen. Im Operationssaal fallen Arbeiten an, deren Durchführung überdacht und besser gelöst werden muss.

Es gilt, die Arbeit von Schwestern und Pflegern so effektiv wie möglich zu gestalten. Auch der Komfort der Arbeit, dem Optimierungsbemühungen gelten müssen, sollte berücksichtigt werden.

Aus diesem Grunde haben wir uns Gedanken gemacht, wie die Schwestern und Pfleger im OP in der präoperativen Vorbereitung entlastet und effektiv eingesetzt werden können.

Wir haben ein Hilfsmittel entwickelt, welches bei Operationen am Fuß und distalen Unterschenkel bis zur Unterschenkelmitte das Halten des Beines durch eine Schwester oder einen Pfleger während der OP-Vorbereitung entbehrlich macht.

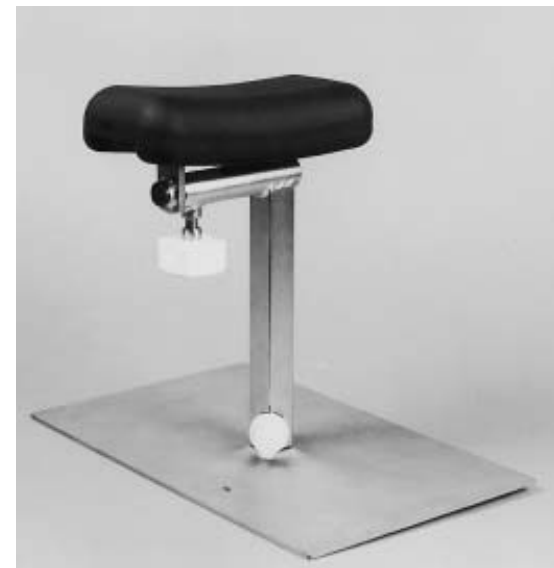

Abb.1 Beinhalter für die präoperative Vorbereitung.

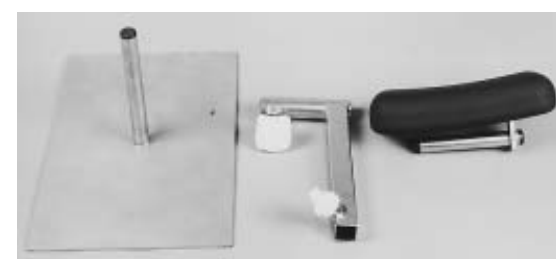

Abb.2 Einzelteile des Beinhalters mit der gekehlten Lagerungsplatte.

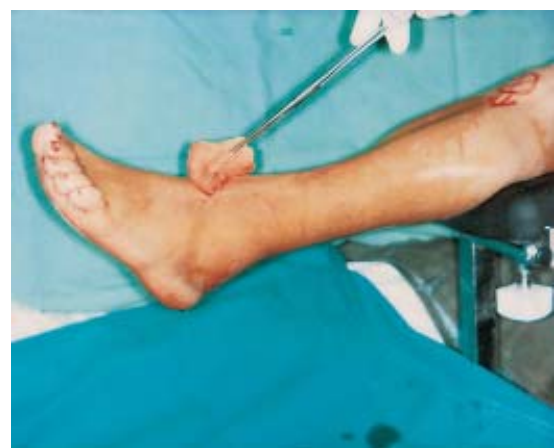

Abb. 3 Beinhalter im Einsatz zur präoperativen Desinfektion des OP-Gebietes am Fuß bis mittleren Unterschenkel.

\footnotetext{
${ }^{*}$ Fa. Maquet, Rastatt, Hr. Winterhalter
}

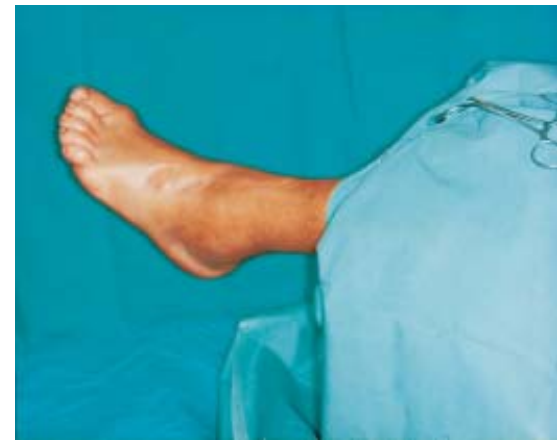

Abb. 4 Der Beinhalter ersetzt eine Pflegekraft bis zur Teilabdeckung.

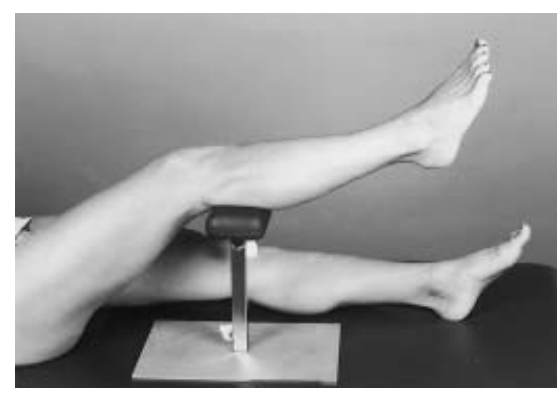

Abb.5 Der Beinhalter kann zur Unterstützung bei Eingriffen an der Unterschenkelrückseite oder an der Ferse eingesetzt werden.

In Zusammenarbeit mit einer Fa. für Lagerungsartikel* entwickelten wir eine Stütze, welche unseres Erachtens diesen Zweck erfüllt. Unser Ziel war es, ein einfach zu handhabendes Gerät zu konstruieren, das möglichst kostengünstig gefertigt werden kann. Für die Konstrukteure bedeutete dies, überwiegend Teile aus der Serienproduktion zu benutzen.

Das Ergebnis unserer gemeinsamen Entwicklungsbemühungen ist die Unterschenkelstütze, die in Abb.1 zu sehen ist. Sie besteht aus einer stabilen, hygienisch einwandfreien Stahlplatte, auf die eine in der Höhe verstellbare Stütze gesteckt werden kann. Die Konstruktion ermöglicht einen Rechts-links-Gebrauch. Auf der Vertikalstütze ist ein Ho- 
rizontalträger aufgesetzt, auf welchem in jeder Position die gepolsterte Lagerungsplatte aus der Serienproduktion fixiert werden kann. Um das Abgleiten des Unterschenkels von der Stütze zu vermeiden, ist die Halterung gekehlt (Abb.2).

\section{Einsatz des Gerätes}

Die Stütze kann als Abwasch- und Desinfektionshilfe bentutzt werden (Abb.3). Bewährt hat sich das Gerät bei unseren Einsätzen insbesondere bei offenen Verletzungen, die eine sorgfältige und aufwendige präoperative Wundreinigung notwendig machen.

Nach der Wundreinigung und Desinfektion kann die Stütze bis zum Zeitpunkt der Teilabdeckung belassen werden (Abb.4). Der sogenannte Springer im OP oder auch das übrige für diese Aufgaben sonst eingesetzte OP-Personal kann in der Zwischenzeit der Instrumentierschwester bei der Vorbereitung des OP-Materials behilflich sein.
In einigen Fällen, bei welchen an der Ferse oder an der Dorsalseite des Unterschenkels operiert werden musste, und eine Bauchlage aus anästhesiologischen Gründen nicht möglich war, konnten wir unter Zuhilfenahme der Stütze bequem in dieser Position operieren (Abb.5).

Dieser Einsatz bleibt sicher Ausnahmefällen vorbehalten.

Bei längerer Lagerung auf der Unterschenkelstütze ist auf eine zusätzliche Polsterung zu achten, um Lagerungsschäden zu vermeiden.

\section{Diskussion}

Wir Ärzte sollten uns bemühen, auch das Arbeitsfeld des Pflegepersonals so angenehm und effektiv wie möglich zu gestalten. Wir sollten dafür Sorge tragen, dass der Personaleinsatz sinnvoll gestaltet wird, ohne dass die Qualität leidet. Es muss unser Ziel sein, wenn irgend mög- lich, Pflegepersonal für einfache Hilfstätigkeiten durch Gerätschaften zu ersetzen. Das hier vorgestellte Gerät ist nach längerem Probeeinsatz in unserer Klinik ein gut funktionierendes Hilfsmittel in der präoperativen Vorbereitung.

\section{Dr. med. S. Rüibenacker}

Kreiskrankenhaus Langenau Karlstr. 45

89129 Langenau 